\title{
Intensive simulation training on urological mini-invasive procedures using Thiel-embalmed cadavers: The IAMSurgery experience
}

\author{
Guglielmo Mantica ${ }^{1,2}$, Giovannalberto Pini ${ }^{2,3}$, Davide De Marchi ${ }^{2,3}$, Irene Paraboschi ${ }^{4}$, \\ Francesco Esperto ${ }^{5}$, André Van der Merwe ${ }^{6}$, Heidi Van Deventer ${ }^{6}$, Massimo Garriboli ${ }^{7}$, \\ Nazareno Suardi ${ }^{1}$, Carlo Terrone ${ }^{1}$, Rosario Leonardi ${ }^{2,3}$ \\ ${ }^{1}$ Department of Urology, Policlinico San Martino Hospital, University of Genova, Genova, Italy; \\ 2 IAMSurgery, International Accademy of Miniinvasive Surgery; \\ ${ }^{3}$ Department of Urology, San Raffaele Turro Hospital, Milan, Italy; \\ ${ }^{4}$ Department of Neuroscience, Rehabilitation, Ophthalmology, Genetics, Maternal and Child Health (DINOGMI), \\ University of Genova, Genova, Italy; \\ ${ }^{5}$ Department of Urology, Campus Bio-medico University of Rome, Rome, Italy; \\ ${ }^{6}$ Department of Urology, Stellenbosch University and Tygerberg Academic Hospital, Cape Town, South Africa; \\ ${ }^{7}$ Department of Pediatric Urology, Evelina Children Hospital, London, UK.
}

\begin{abstract}
Summary Introduction: The objective of the study was to evaluate the benefits perceived by the use of cadaver models by IAMSurgery attendees and to define indications to standardize future similar training camps.

Materials and methods: A 25-item survey was distributed via $e$-mail to all the participants of previous training courses named as "Urological Advanced Course on Laparoscopic Cadaver Lab" held at the anatomy department of the University of Malta, for anonymous reply. Participants were asked to rate the training course, the Thiel's cadaveric model, and make comparison with other previously experienced simulation tools.

Results: The survey link was sent to 84 attendees, with a response rate of $47.6 \%$ (40 replies). There was improvement in the median self-rating of the laparoscopic skills before and after the training camp with a mean difference of $0.55 / 5$ points in the post-training skills compared to the basal $(p<0.0001)$. The $72.2 \%$ of the urologists interviewed considered Thiel's HCM better than other training methods previously tried, while five urologists $(27.8 \%)$ considered it equal ( $p=0.00077)$. Globally, $77.5 \%$ (31) of attendees found the training course useful, and $82.5 \%$ (33) would advise it to colleagues. Conclusions: Thiel's fixed human cadaveric models seem to be ideal for training purposes, and their use within properly structured training camps could significantly improve the surgical skills of the trainees. An important future step could be standardization of the training courses using cadavers, and their introduction into the standardized European curriculum.
\end{abstract}

KEY WORDS: Training; Cadaver model; Urology; Thiel fixation; Simulation.

Submitted 22 November 2019; Accepted 7 December 2019

\section{INTRODUCTION}

Surgical ethics requires that surgical procedures should be performed on patients only after having reached adequate skills that ensure high standards in terms of quality and safety for the patient himself $(1,2)$. For this reason, in recent years, we have witnessed the multiplica- tion of theoretical, technological, synthetic, and biological simulation/training models of varying utility for urologists and post-graduates (3-7). Although often useful, many of these models have significant limitations in terms of realism, accessibility, and ethics. As trivial as it sounds, nothing is more like the human body as the human body itself. For this reason, a solution in terms of education and pre-real patient surgical training could be provided by increased use of human cadaver models. Currently, there is little (but increasing) literature on the use of human cadaveric models (HCM) for training purposes, and there are a few experiences of training camps with a standardized format reported (8-14).

The IAMSurgery has been organizing laparoscopic urological surgery training camps for several years using human cadaveric models. The objective of the study is, therefore, to evaluate the benefits perceived by the use of cadaver models by IAMSurgery attendees and to define indications to standardize future similar training camps.

\section{Materials AND MEthods}

From January 2016 to October 2019, the IAMSurgery (International Academy of Mini-invasive Surgery) organized six editions of a training camp called "Urological Advanced Course on Laparoscopic Cadaver Lab".

The courses were held at the anatomy department of the University of Malta, an expert in the advanced preparation of the bodies following the Thiel's soft-fix embalming method.

Preparation of the Thiel's cadaveric models The "Thiel method" 15 consists of the application of an intravascular injection formula, and submersion for a determinate time in a stainless steel tank in a particular solution that lacks toxic or irritating gases due to minimum formaldehyde concentrations. Thiel fixation provides "reusable" cadavers on which, in some cases, several proce-

No conflict of interest declared. 
dures might be performed, being more cost-effective than fresh and fresh frozen cadavers.

\section{Format of the course}

The course starts with six hours of face-to-face interactive lectures on embalming technique, preparation of the corpses, pelvic and retroperitoneal anatomy, patient positioning, followed by step-by-step modular videos on pelvic and kidney surgery. The hands-on practice began early on the second day. The course, supported by the expert faculty, allows practising simultaneously on three cadavers for a total time of 24 hours. Two four-hour modules were focused on laparoscopic radical prostatectomy, two four-hour modules on laparoscopic partial nephrectomy, and two four-hour modules on laparoscopic radical nephrectomy. The philosophy of the course was to maintain small groups for each procedure, favoring a modular rotation regulated by the tutor, to teach not only surgical technique but also non-technical skills and encouraging the team building, a fundamental requirement in the operating room and real life.

\section{Study design and data analysis}

A 25-item survey (Table 1) was designed by two of the course tutors (R.L. and G.P.) and checked by a third urologist (G.M.) not previously involved in the organization of the courses. The survey was designed following the Checklist for Reporting Results of Internet E-Surveys (CHERRIES) guidelines, 16 uploaded on Google Form, and was distributed via e-mail to all the participants of the previous courses, for anonymous reply. The survey was distributed in November 2019. Before circulating, we tested the survey for usability and technical functionality.

The survey consists of two parts:

- Part one: General information of the responder (including age, trainee vs. specialist, nationality).

- Part two: Ratings of the training course, the Thiel's cadaveric model, and comparison with other previously experienced simulation tools.

Some questions had a free answer option while others a ranking scale from 1 (very low) to 5 (very high). Only surveys with section one completed, and more than 90\% completed in section two, were included in the analysis. Data were entered into a Microsoft Excel (version 14.0) database and transferred to SofastatsTM for Windows.

A descriptive statistical analysis was performed. Variables are presented as median ( $1^{\text {st }}-3^{\text {rd }}$ interquartile range) or as a percentage (\%).

The statistical analysis of nominal variables was done using the T-Test Calculator and the Chi-Square Calculator for Goodness of Fit. The level of significance was set at $\mathrm{p}<0.05$.

\section{Table 1.}

IAMSurgery - Malta - Training course on Thiel cadaver models.
1) Age
2) Working position at the time of the training course
3) Nationality
4) Were you already performing laparoscopy before the training camp? (YES/NO)
5) If NO; Have you started to perform laparoscopy after the training camp? (YES/NO)
6) Please give a mark to the team of Tutors (1 to 5 )
7) Please give a mark to the Course Concept (1 to 5$)$
8) Please give a mark to the Time planning (was the training course too short/long?) (1 to 5)
9) Usefulness of cadaver training model for radical nephrectomy (1 to 5 )
10) Realism of cadaver training model for radical nephrectomy (1 to 5 )
11) Usefulness of cadaver training model for partial nephrectomy (1 to 5)
12) Realism of cadaver training model for partial nephrectomy (1 to 5 )
13) Usefulness of cadaver training model for prostatectomy (1 to 5 )
14) Realism of cadaver training model for prostatectomy (1 to 5)
15) Do you have any experience with other training models (i.e. porcine, synthetic, etc)? (YES/NO)
16) If YES; please state what other type of training model have you tried
17) If YES; please explain the differences/advantages/disadvantages of the cadaveric model com- pared to the others that you have experienced (i.e. any difference in bleeding?)
18) How would you rate the Thiel cadaveric model? (1 to 5)
19) If you do have experiences with other training models, how do you consider Thiel cadaver model? (worse-equal-better)
20) Please rate your laparoscopic skills confidence before the training (1 to 5 )
21) Please rate your laparoscopic skills confidence after the course (1 to 5 )
22) Were your couse expectations fullfilled? (YES/NO/NOT COMPLETELY)
23) Have you found the training camp useful for your clinical and surgical practice? (YES/NO/NOT COMPLETELY)
24) How would you rate the training course? (1 to 5 )
25) Would you advice it to a colleague? (YES/NO/I DON'T KNOW)

\section{REsULTS}

The survey link was sent to 84 attendees, with a response rate of $47.6 \%$ (40 replies).

All attendees were Italian, specialized in urology, and with a median age of 50 years (4357.5). Twenty-seven (67.5\%) were already performing laparoscopy at their institution (either as first operator or assistant). Ten (76.9\%) out of the 13 who were not performing laparoscopy started with this minimally invasive approach after the training course. The ratings given to the training course and the usefulness and realism of the HCM for training purposes are summarized in Table 2. There was improvement in the median selfrating of the laparoscopic skills before and after the training camp with a mean difference of $0.55 / 5$ points in the post-training skills compared to the basal ( $\mathrm{p}<0.0001$ ).

Twenty-one (52.5\%) attendees did not have experience with any other simulator models, while $18(45 \%)$ had tried at least one other method (one surveyed colleague did not reply to this question). Porcine and virtual models were both tried by eight urologists while a synthetic model was tried by five urologists. The presence of bleeding was stated in favor of porcine models by seven urologists $(87.5 \%)$ while the realism of anatomy $13(72.2 \%)$ and better tissue consistency 8 (44.4\%) was in favor of Thiel's HCM. Thirteen (72.2\%) urologists considered Thiel's HCM better than other training meth- 
Table 2.

Overall and specific course rating.

\begin{tabular}{|lccccc}
\hline Variable: respondent numbers (\%) & $\mathbf{1}$ & $\mathbf{2}$ & $\mathbf{3}$ & $\mathbf{4}$ & $\mathbf{5}$ \\
\hline Tutors rating & - & - & $1(2.5 \%)$ & $13(32.5 \%)$ & $26(65 \%)$ \\
\hline Course concept rating & - & - & $1(2.5 \%)$ & $6(15 \%)$ & $33(82.5 \%)$ \\
\hline Time planning rating & - & - & $8(20 \%)$ & $16(40 \%)$ & $16(40 \%)$ \\
\hline Useful of HCM for RN & - & - & - & $10(25 \%)$ & $30(75 \%)$ \\
\hline The realism of HCM for RN & - & - & $2(5 \%)$ & $19(47.5 \%)$ & $19(47.5 \%)$ \\
\hline Useful of HCM for PN & - & $2(5 \%)$ & $8(20 \%)$ & $20(50 \%)$ & $10(25 \%)$ \\
\hline The realism of HCM for PN & - & $4(10 \%)$ & $12(30 \%)$ & $18(45 \%)$ & $6(15 \%)$ \\
\hline Useful of HCM for RP & - & - & $7(17.5 \%)$ & $17(42.5 \%)$ & $16(40 \%)$ \\
\hline The realism of HCM for RP & - & - & $6(15 \%)$ & $17(42.5 \%)$ & $17(42.5 \%)$ \\
\hline Thiel HCM rating & - & - & $3(7.5 \%)$ & $21(52.5 \%)$ & $16(40 \%)$ \\
\hline Pre-course personal LAP skills & $8(20 \%)$ & $6(15 \%)$ & $16(40 \%)$ & $10(25 \%)$ & - \\
\hline Post-course personal LAP skills & - & $8(20 \%)$ & $14(35 \%)$ & $18(45 \%)$ & - \\
\hline Overall course rating & - & - & $3(7.5 \%)$ & $16(40 \%)$ & $21(52.5 \%)$ \\
\hline
\end{tabular}

Figure 1.

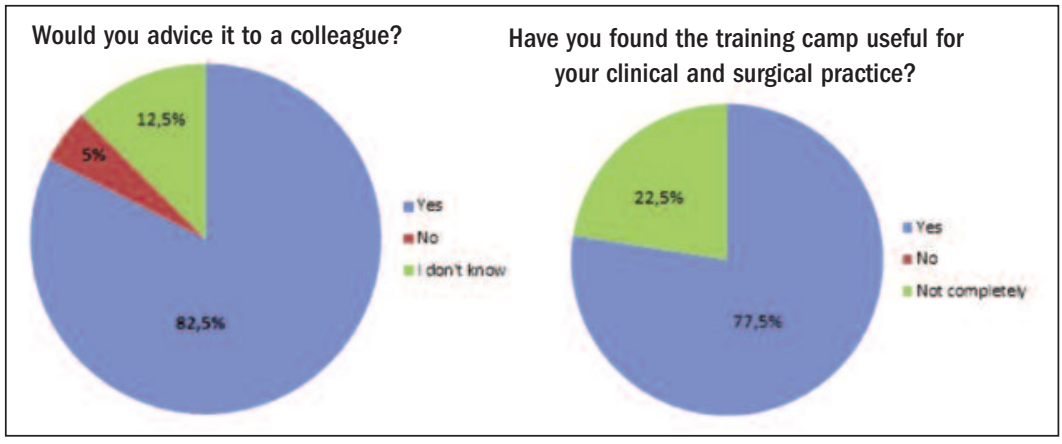

training purposes (8). The Thiel method provides cadavers that can be re-used and on which many procedures can be performed.

The re-usability is of paramount importance, considering the scant supply of human bodies available for research and training in some settings.

Moreover, from our survey, it emerges how most of the interviewees consider the cadaver model globally superior compared to the other tested models (porcine, synthetic, and virtual).

The only flaw is the absence of bleeding, which compromises complete realism in particular in some procedures such as partial nephrectomy.

Similarly, the Thiel method has already been tested in urology and showed to be suitable for training and testing purposes within minimally-invasive approaches (19-20)

Surgical training in adult cadaveric models may be useful also for pediatric urologists regarding some specific procedures such as nephrectomies (i.e., performed for Wilms 'tumors).

The anatomy of an infant is different; however, a teenager often presents with an anatomy similar to an adult.

Furthermore, while taking into account the limits as mentioned earlier, the

ods previously tried, while five urologists (27.8\%) considered it equal $(\mathrm{p}=0.00077)$.

Globally, $77.5 \%$ (31) of attendees found the training course useful, and $82.5 \%$ (33) would advise it to colleagues (Figure 1).

\section{Discussion}

Surgical training is very delicate and for ethical reasons cannot be performed directly on the patient but requires a structured modular training first in the dry lab, then on animal or cadaveric models (17)

The "Urological Advanced Course on Laparoscopic Cadaver $L a b "$ is a three-day training camp that combines theory, surgical practice, and team building.

The cadaveric model is designed to bridge the gap between simulation and live surgery. In literature, there are other training camp reports on cadavers in different fields of urology with excellent feedback from the participants who generally perceive an improvement in their operating skills at the end of the course itself (10-14).

In such courses, the importance of the tutors is fundamental (18).

The preparation, the ability to teach, and the passion of an excellent tutor can affect the quality of the contents.

The quality of the cadaver models is also fundamental. Due to biological risk, human cadavers are often used after an embalming process (15). The most common method of embalming is formalin fixation. However, a new method called "Thiel fixation" provides an alternative to fresh or formalin-fixed specimens and can be ideal for pediatric surgeon could benefit from confidence-building with tissue consistency and surgical planning.

From an educational point of view, Thiel's model might be a perfect tool to be introduced into standardized European curricula for urologists and pediatric urologists.

The future perspective of IAMS is to make the training even more realistic by mimicking a real surgical environment through a live cadaver model, and the anatomy department of the University of Malta is already at work to provide a cadaver perfusion system. The model will combine the realistic conditions of the living body with the real human anatomy in one model and is the only training model available that provides such a combination $(21,22)$.

\section{Conclusions}

Thiel's fixed human cadaveric models seem to be ideal for training purposes, and their use within properly structured training camps could significantly improve the surgical skills of the trainees. An important future step could be standardization of the training courses using cadavers, and their introduction into the standardized European curriculum.

\section{ACKNOWLEDGements}

A kind and sincere thank you to Dr. Heidi Van Deventer for the English editing. 


\section{References}

1. Carrion DM, Rodriguez-Socarrás ME, Mantica G, et al. Current status of urology surgical training in Europe: an ESRU-ESU-ESUT collaborative study. World J Urol. 2019 Apr 13.

2. de Oliveira TR, Cleynenbreugel BV, Pereira S, et al. Laparoscopic training in urology residency programs: a systematic review. Curr Urol. 2019; 12:121-126.

3. Tawfik AM, El-Abd AS, El-Enen MA, et al. Validity of a sponge trainer as a simple training model for percutaneous renal access. Arab J Urol. 2017; 15:204-210.

4. Monda SM, Weese JR, Anderson BG, et al. Development and validity of a silicone renal tumor model for robotic partial nephrectomy training. Urology. 2018; 114:114-120.

5. Mantica G, Pacchetti A, Aimar R, et al. Developing a five-step training model for transperineal prostate biopsies in a naïve residents' group: a prospective observational randomised study of two different techniques. World J Urol. 2019; 37:1845-1850.

6. Setia S, Feng C, Coogan C, et al. Urology residents' experience with simulation: initial evaluation of MRI/US fusion biopsy workshop. Urology 2019; pii: S0090-4295(19)30817-9.

7. Mantica G, Balzarini F, Dotta F, et al. Development of a photographic handbook to improve cystoscopy findings during resident's training: A randomised prospective study. Arab J Urol. 2019; $17: 243-248$

8. Mantica G, Leonardi R, Pini G, et al. The current use of human cadaveric models in urology: a systematic review. Minerva Urol Nefrol. 2019 Nov 11. doi: 10.23736/S0393-2249.19.03558-6.

9. Healy SE, Rai BP, Biyani CS, et al. Thiel embalming method for cadaver preservation: a review of new training model for urologic skills training. Urology. 2015; 85:499-504.

10. Yiasemidou M, Roberts D, Glassman D, et al. A multispecialty evaluation of Thiel cadavers for surgical training. World J Surg. 2017; 41:1201-1207.

11. Mains E, Tang B, Golabek T, et al. Ureterorenoscopy training on cadavers embalmed by Thiel's method: simulation or a further step towards reality? Initial report. Cent European J Urol. 2017; 70:81-87.

12. Bele U, Kelc R. Upper and Lower Urinary Tract Endoscopy Training on Thiel-embalmed Cadavers. Urology. 2016; 93:27-32.

13. Özan S, Huri E, Tatar I, et al. Impact of cadaveric surgical anatomy training on urology residents knowledge: a preliminary study. Turk J Urol. 2015; 41:83-7.

14. Ahmed K, Aydin A, Dasgupta P, et al. A novel cadaveric simulation program in urology. J Surg Educ. 2015; 72:556-65.

15. Thiel $W$. The preservation of the whole corpse with natural color. Ann Anat. 1992; 174:185-95.

16. Eysenbach G. Improving the quality of Web surveys: the Checklist for Reporting Results of Internet E-Surveys (CHERRIES). J Med Internet Res. 2004; 6:e34.

17. Somani BK, Van Cleynenbreugel B, Gozen A, et al. The European Urology Residents Education Programme hands-on training format: 4 years of hands-on training improvements from the European School of Urology. Eur Urol Focus. 2019; 5:1152-1156.

18. Mantica G, Fransvea P, Virdis F, et al. Surgical training in South Africa: an overview and attempt to assess the training system from the perspective of foreign trainees. World J Surg. 2019; 43:2137-2142.

19. Rai BP, Stolzenburg JU, Healy S, et al. Preliminary validation of Thiel embalmed cadavers for laparoscopic radical nephrectomy. $J$ Endourol. 2015; 29:595-603.

20. Veys R, Verpoort P, Van Haute C, et al. Thiel-embalmed cadavers as a novel training model for ultrasound guided supine endoscopic combined intrarenal surgery. BJU Int. 2019 Nov 16. doi: 10.1111/bju.14954. [Epub ahead of print]

21. Aboud ET, Aboud G, Aboud T. "Live cadavers" for practicing airway management. Mil Med. 2015; 180 (3 Suppl):165-70.

22. Held JM, McLendon RB, McEvoy CS, Polk TM. A reusable perfused human cadaver model for surgical training: an initial proof of concept study. Mil Med. 2019;184(Suppl 1):43-47.

\section{Correspondence}

Guglielmo Mantica, MD (Corresponding Author) guglielmo.mantica@gmail.com

Department of Urology, Policlinico San Martino Hospital, University of Genova Largo Rosanna Benzi 10, 16132, Genova (Italy)

Giovannalberto Pini, MD

Davide De Marchi, MD

Rosario Leonardi, $M D$

Department of Urology, San Raffaele Turro Hospital, Milan (Italy)

Irene Paraboschi, MD

Department of Neuroscience, Rehabilitation, Ophthalmology, Genetics, Maternal and Child Health (DINOGMI), University of Genova, Genova (Italy)

Francesco Esperto, MD

Department of Urology, Campus Bio-medico University of Rome, Rome (Italy)

André Van der Merwe, MD

Department of Urology, Stellenbosch University and Tygerberg Academic Hospital, Cape Town (South Africa)

Massimo Garriboli, MD

Department of Pediatric Urology, Evelina Children Hospital, London (UK)

Nazareno Suardi, MD

Carlo Terrone, $M D$

Department of Urology, Policlinico San Martino Hospital, University of Genova, Genova (Italy) 